\title{
Evaluation of Vermicularity of Compacted Graphite Iron Based on Multiple Characteristic Points of Thermal Analysis
}

\author{
Ailong Jiang ${ }^{1,2}$, Xuelei Tian ${ }^{1}$, Hongda Song ${ }^{3}$, Guili $\mathrm{Gao}^{2,3}$, Qiang $\mathrm{Wu}^{4}$, Dequan $\mathrm{Shi}^{3}$, \\ Zhenyu $\mathrm{Xu}^{3, *}$ and Yubin $\mathrm{Wu}^{4}$ \\ ${ }^{1}$ School of Materials Science and Engineering, Shandong University, Jinan 250061, Shandong, China \\ ${ }^{2}$ Weichai Power Co., Ltd., Weifang 261061, Shandong, China \\ ${ }^{3}$ School of Materials Science and Engineering, Harbin University of Science and Technology, Harbin 150040, Heilongjiang, China \\ ${ }^{4}$ Harbin Kedewei Metallurgy Co., LTD., Harbin 150078, Heilongjiang, China
}

The vermiculation effects of compacted graphite cast iron are seriously affected by the metallurgical and technological factors. The change of characteristic points on the cooling curve is attributed to the interaction of these factors, so it is difficult to establish the evaluation relationship between the vermicularity and the characteristic points of thermal analysis curve. Based on the idea of statistics, a method was studied to evaluate the vermicularity of molten iron by comparing with an established characteristic points representation model of hypothetical ideal metallurgical quality state under the production condition. The results showed that the method can meet the requirements of evaluating the vermicularity of molten iron by multiple characteristic points on the cooling curve. As the matching process among cooling curves was omitted, the calculation and evaluation efficiency can be improved. In addition, it is expected to achieve on-line classification and regulation of vermiculation effect in front of furnace by expanding the sample capacity for establishing the characterization model.

[doi:10.2320/matertrans.MT-M2020356]

(Received November 24, 2020; Accepted February 10, 2021; Published March 12, 2021)

Keywords: compacted graphite iron, vermicularity evaluation, thermal analysis, multiple characteristic points, metallurgical characteristics

\section{Introduction}

Compacted graphite iron (CGI) contains graphite particles in compacted/vermicular form in steel like matrix, of which the graphite shape is an intermediate morphology between flake and spheroidal graphite. Thus, it includes an optimal combination of different properties like strength, ductility and thermal conductivity of other cast iron types. ${ }^{1,2)}$ Because of these attractive properties, difference grades of CGI have been proved valuable in many applications such as exhaust manifolds, hydraulic valves, diesel engine blocks and machine parts during recent years. ${ }^{3,4)}$

An acceptable CGI should contain less than $20 \%$ spheroidal graphite and more than $80 \%$ compacted form graphite, without any flake graphite which can cause local weaknesses and lead to failure. ${ }^{5)}$ As we all know, the technological window of CGI production is very narrow, and the fading effects should be considered. Therefore, the initial starting status of the molten iron should be held far away from the flake graphite region. And in order to prevent large amounts of nodule graphite, the best pouring position is between the compacted graphite and spheroidal graphite region. During holding, the as-cast microstructure will reach the compacted graphite stable platform, with no flake graphite and no more 20 percent spheroidal graphite in matrix. ${ }^{6)}$ In addition, the oxygen and sulphur content and amount of inoculants may shift the CGI technological platform. ${ }^{7,8)}$

Nowadays, several casting methods of CGI have been developed, trying to find a precise control method for the final microstructure. ${ }^{5,6)}$ Thermal analysis is the reflection of a solidification history of its related sample in which each phase transformation causes a thermal event on its cooling curve. ${ }^{9)}$ The characteristic temperatures on the cooling curve

*Corresponding author, E-mail: zhenyuxu2006@126.com are called "metallurgical fingerprint" of molten iron metallurgical state. However, it is difficult to distinguish compacted graphite iron and ductile iron by comparing the characteristic temperatures in thermal analysis. ${ }^{10)}$

Li et al. ${ }^{11-14)}$ developed a correlating method which evaluates the melt quality of ductile iron (DI) and CGI using the freezing region from liquidus to solidus temperature in the cooling curve and the computer database technology. When the difference between the cooling curves of a molten iron to be measured and the melt whose metallurgy state is known is lower than the threshold, the same graphite shape exists under the same solidification condition. However, due to the large number of temperature points collected in the solidification section in the cooling curve, and with the increase of sample cooling curve in the database, the matching time of the curve will be too long. This leads to the decline of inoculation and vermiculation effect of the molten iron, and reduces the practicability of the production practice. In this paper, a multi-characteristic-point representation model was established for a hypothetical molten iron with ideal metallurgical quality state, which is used as a reference to identify the measured cooling curve and evaluate the vermiculation effect of the treated molten iron. The results showed that the matching time of the measured curve can be sufficiently shortened and the vermiculation identification result can be achieved well.

\section{Materials and Experiments}

\subsection{Experimental procedure}

The experiments were made in Binhai Foundry, Weichai Heavy Machinery Co., Ltd. with a medium frequency induction furnace. The charging materials mainly consist of pig iron, scrap steel, returns, carbon riser, various intermediate alloys etc. When the charging materials were 
Table 1 Chemical compositions of molten iron in experiments, mass $\%$.

\begin{tabular}{|c|c|c|c|c|c|c|c|c|c|c|}
\hline Molten iron & $\mathrm{C}$ & $\mathrm{Si}$ & $\mathrm{Mn}$ & $\mathrm{P}$ & S & $\mathrm{Cu}$ & Sn & $\mathrm{Mg}$ & RE & $\mathrm{CE}^{*}$ \\
\hline Base iron & $3.60 \sim 3.80$ & $1.20 \sim 1.50$ & $0.30 \sim 0.50$ & $\leq 0.05$ & $0.01 \sim 0.02$ & $0.30 \sim 0.60$ & $0.02 \sim 0.04$ & - & - & $4.20 \sim 4.32$ \\
\hline Post-treated & $3.70 \sim 3.80$ & $2.00 \sim 2.40$ & $0.30 \sim 0.50$ & $\leq 0.05$ & $0.01 \sim 0.02$ & $0.30 \sim 0.60$ & $0.02 \sim 0.04$ & $0.01 \sim 0.02$ & $0.01 \sim 0.02$ & $4.38 \sim 4.62$ \\
\hline
\end{tabular}

Table 2 Chemical compositions of the furnace charge and various treating agents, mass $\%$.

\begin{tabular}{ccccccccc}
\hline Materials & $\mathrm{Mg}$ & $\mathrm{RE}$ & $\mathrm{Si}$ & $\mathrm{Ca}$ & $\mathrm{Al}$ & $\mathrm{MgO}$ & $\mathrm{Ba}$ & $\mathrm{Fe}$ \\
\hline $\begin{array}{c}\text { Vermiculation } \\
\text { wire of } \Phi 13 \mathrm{~mm}\end{array}$ & $7.0 \sim 10.0$ & $3.0 \sim 6.0$ & $43.0 \sim 45.0$ & $2.0 \sim 5.0$ & - & $\leq 2.0$ & - & Bal. \\
$\begin{array}{c}\text { Inoculation wire } \\
\text { of } \Phi 13 \mathrm{~mm}\end{array}$ & - & $1.0-1.5$ & $54.0-56.0$ & $2.0-2.5$ & $0.5 \sim 1.0$ & $\leq 2.0$ & $5.0-6.0$ & Bal. \\
\hline
\end{tabular}

totally melted down and the temperature had risen to over $1500^{\circ} \mathrm{C}$, a sample was poured for $\mathrm{C}, \mathrm{Si}$ and $\mathrm{CE}$ check by thermal analysis. Simultaneously, a chilled sample was poured for chemical analysis by the emission spectrometry, and $\mathrm{C}$ and $\mathrm{S}$ content determination by the combustion method. The composition of molten iron was controlled according to Table 1.

Treatment was made by the wire feeding method in a 0.5 ton ladle. The types and specifications of vermicularizing agent and inoculant cored wires are shown in Table 2. After each treatment, the molten iron was sampled and poured into a cup with inside size of $30 \times 50 \mathrm{~mm}$. The cooling temperature data were recorded by a set of data collector. After cutting, mounting and polishing, the thermal analysis samples near the hot node of the cups were investigated by the optical microscope. Image analysis were then performed on the samples in order to define the amount of vermicularity by image-Pro Plus software.

\subsection{Characteristic points correlation with solidification on the cooling curve of CGI}

Cooling curve of cast iron during solidification reveals various peculiarities which can indicate some information on the sample metallurgy state. Before the precipitation of crystalline phase, the temperature of molten iron decreases at a constant rate from the maximum temperature $T_{\max }$. When the liquidus temperature $T_{\mathrm{L}}$ is reached, the primary austenite will nucleate and continuously release the latent heat of crystallization, resulting in the slow cooling rate. At the eutectic stage, the highest cooling rate $(\mathrm{d} T / \mathrm{d} t)_{\mathrm{N}}$ of eutectic nucleation appears on the differential curve, corresponding to the eutectic nucleation temperature $T_{\mathrm{N}}$ on the cooling curve. Due to the undercooling, the eutectic austenite precipitates and release a large amount of latent heat. So the cooling rate drops rapidly to zero and the temperature drops to the minimum eutectic temperature $T_{\mathrm{EU}}$. After that the temperature will rise reversely. The simultaneous growth of eutectic

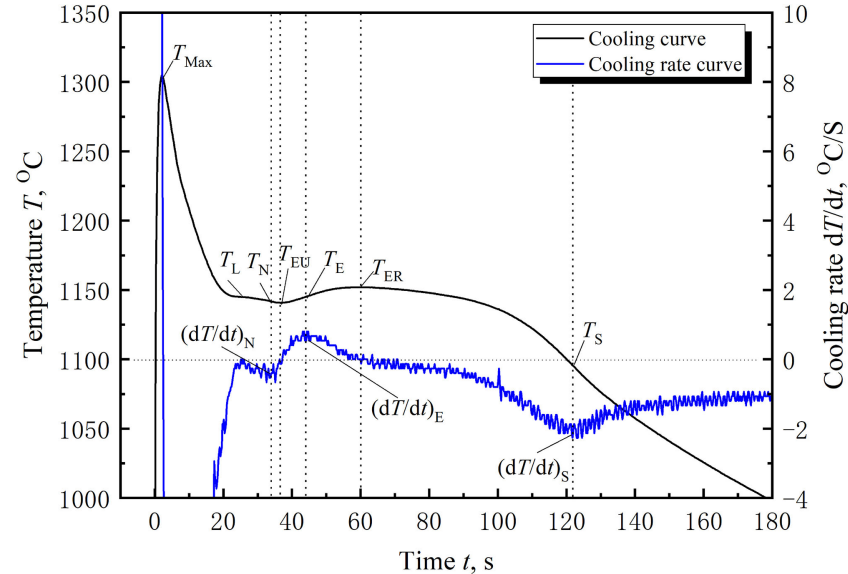

Fig. 1 Illustration of 10 critical characteristic points of vermicularity evaluation of CGI: 7 temperature points of the cooling curve and 3 first order differential points on corresponding cooling rate curve.

austenite and graphite will release more latent heat of crystallization, and the maximum eutectic recovery rate $(\mathrm{d} T / \mathrm{d} t)_{\mathrm{E}}$ is arrived, which corresponds to temperature $T_{\mathrm{E}}$ on the cooling curve. After the maximum eutectic recovery rate, the eutectic growth rate and the released latent heat of crystallization decreases. However, the temperature rises until the maximum eutectic recovery temperature $T_{\mathrm{ER}}$, and then the temperature begins to decrease again. When the minimum value $(\mathrm{d} T / \mathrm{d} t)_{\mathrm{S}}$ on differential curve is reached, the molten iron completely solidified at the corresponding temperature $T_{\mathrm{S}}$. Then the solid-state phase transition occurs.

The 10 critical characteristic points are revealed in Fig. 1, and the descriptions of the nomenclature are listed in Table 3. As mentioned in Refs. 10, 11), they comprehensively express the position and shape of the solidification section of the cooling curve. Therefore, the combination of these 10 points should be able to represent the metallurgy quality state characteristics of the molten iron. 
Table 3 Nomenclature and definition of the critical characteristic points on a CGI cooling curve and its first derivative.

\begin{tabular}{cl}
\hline Designation & \\
\hline$T_{\mathrm{Max}},{ }^{\circ} \mathrm{C}$ & Maximum temperature \\
$T_{\mathrm{L}},{ }^{\circ} \mathrm{C}$ & Liquification \\
$T_{\mathrm{N}},{ }^{\circ} \mathrm{C}$ & Temperature at eutectic nucleation \\
$(d T / d t)_{\mathrm{N}},{ }^{\circ} \mathrm{C} / \mathrm{s}$ & Highest cooling rate at eutectic nucleation \\
$T_{\mathrm{EU}},{ }^{\circ} \mathrm{C}$ & Minimum eutectic temperature \\
$T_{\mathrm{E}},{ }^{\circ} \mathrm{C}$ & Temperature at maximum eutectic recovery rate \\
$(d T / d t)_{\mathrm{E}},{ }^{\circ} \mathrm{C} / \mathrm{s}$ & Maximum eutectic recovery rate \\
$T_{\mathrm{ER}},{ }^{\circ} \mathrm{C}$ & Maximum temperature at eutectic recovery \\
$T_{\mathrm{S}},{ }^{\circ} \mathrm{C}$ & Temperature at end of solidification \\
$(d T / d t)_{\mathrm{S}},{ }^{\circ} \mathrm{C} / \mathrm{s}$ & Cooling rate at end of solidification \\
\hline
\end{tabular}

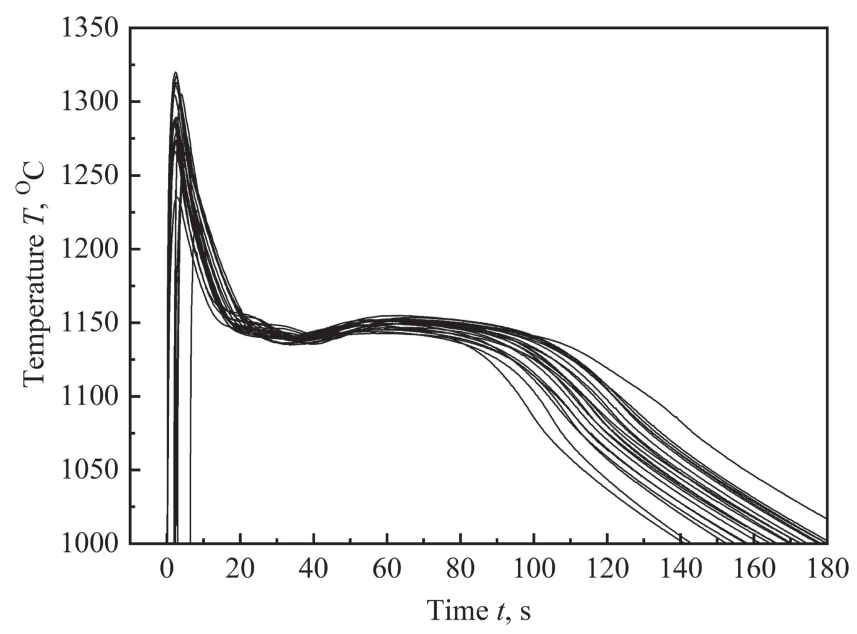

Fig. 222 measured cooling curves of CGI under actual production conditions.

\section{Results and Discussion}

\subsection{Thermal analysis evaluation model of vermiculation effect of CGI}

Figure 2 shows 22 cooling curves of CGI under actual production conditions, and their mainly characteristic point values and vermicularity $V_{\mathrm{G}}$ from metallographic observation are shown in Table 4. From Fig. 2, it can be seen that it takes about 2.5 minutes from pouring to the completion of solidification for the molten iron sample, and are nearly hypoeutectic solidification morphology. The liquidus temperature $T_{\mathrm{L}}$ range is from $1135.1^{\circ} \mathrm{C}$ to $1141.5^{\circ} \mathrm{C}$. The maximum liquidus temperature difference is $6.4^{\circ} \mathrm{C}$ with a small fluctuation. This shows that the carbon equivalent level of the daily molten iron was well controlled. However, the temperature difference from the liquidus temperature $T_{\mathrm{L}}$ to the minimum eutectic temperature $T_{\mathrm{EU}}$ is from $0^{\circ} \mathrm{C}$ to $17.6^{\circ} \mathrm{C}$. It can be seen that the tendency of undercooling of molten iron for eutectic reaction fluctuates greatly, that is to say, the potential energy of graphite nucleation in molten iron is quite different. And this will lead to a great difference in the type and quantity of dendrite growth of pre-eutectic austenite, which will affect the morphology of eutectic cell nucleation and growth. The recalescence temperature $\Delta T_{\mathrm{R}}=T_{\mathrm{ER}}-T_{\mathrm{EU}}$ range of eutectic reaction is from $4.3^{\circ} \mathrm{C}$ to $16.1^{\circ} \mathrm{C}$, and the temperature difference range can reach $11.8^{\circ} \mathrm{C}$. The larger difference of eutectic solidification process will lead to the different appearance of vermicular graphite. Through metallographic examination of the thermal spot center of samples with the different eutectic recalescence temperature, the graphite vermicularity changes between $70 \%$ and $90 \%$, as seen in Table 4. The reason of obvious fluctuation of eutectic recalescence may be related to the initial undercooling fluctuation of molten iron, also including the overlapping influence of other process factors, and there is no direct correlation between them.

Because of the influence of many factors, the characteristic values on the cooling curve vary in a certain range. However, the metallurgical quality state of the molten iron is a combination of multiple parameters, some metallurgical parameters need to be adjusted only when the final vermiculation effect of molten iron is unqualified. The 10 characteristic points on cooling curve shown in Table 4 can basically represent the change trend of the cooling curve. Thus, taking the 10 mean values of every characteristic parameter on cooling curve in Table 4 as a group to represent the ideal metallurgical state of the hypothetical molten iron, which is used as a reference state for the evaluation of metallurgical quality state of practical measured molten iron in front of the furnace. The difference degree parameter $S$ between the 10 corresponding characteristic points on the cooling curve of the molten iron to be measured and the characteristic points representing the hypothetical ideal metallurgical state of molten iron is used to evaluate the actual metallurgical quality state of molten iron. And the calculation formula of difference degree parameter $S$ is as follows:

$$
\left\{\begin{array}{l}
S=\left[\sum \frac{\left(\Delta P_{i}-\Delta P\right)^{2}}{9}\right]^{\frac{1}{2}} \quad i=1,2, \cdots, 10 \\
\Delta P_{i}=P_{i}-P_{R i} \\
\Delta P=\sum \frac{P_{i}-P_{R i}}{10}
\end{array}\right.
$$

Where $P_{i}$ is the characteristic point $i$ on the cooling curve of the measured molten iron corresponding to Table 4, and $P_{R i}$ is the characteristic point $i$ of the hypothetical ideal metallurgical quality state in Table 4 . According to the difference degree parameter $S$, the vermiculation effect of measured molten iron can be judged.

\subsection{Evaluation effect of multiple characteristic point thermal analysis model}

According to formula (1), the difference degree parameter $S$ between the measured data and the hypothetical ideal metallurgical state parameter values in Table 4, and the vermicularity difference can be got. Figure 3 shows the relationship of the difference degree parameter $S$ and vermicularity difference of CGI. From the Fig. 3, it can be seen that there is no obvious regression relationship between 
Table 4 Mainly characteristic point values and vermicularity corresponding to the 22 cooling curves.

\begin{tabular}{|c|c|c|c|c|c|c|c|c|c|c|c|}
\hline \multirow[b]{2}{*}{ Sample No. } & \multicolumn{10}{|c|}{ Characteristic point $P_{i}$} & \multirow{2}{*}{$\begin{array}{l}V_{\mathrm{G}}, \\
\%\end{array}$} \\
\hline & $T_{\text {Max }},{ }^{\circ} \mathrm{C}$ & $T_{\mathrm{L}},{ }^{\circ} \mathrm{C}$ & $T_{\mathrm{N}},{ }^{\circ} \mathrm{C}$ & $\begin{array}{c}(d T / d t) \mathrm{N} \\
{ }^{\circ} \mathrm{C} / \mathrm{s}\end{array}$ & $\boldsymbol{T}_{\mathrm{EU}},{ }^{\circ} \mathrm{C}$ & $T_{E},{ }^{\circ} \mathbf{C}$ & $\begin{array}{c}(d T / d t) \mathrm{E} \\
{ }^{\circ} \mathrm{C} / \mathrm{s}\end{array}$ & $T_{\mathrm{ER}},{ }^{\circ} \mathrm{C}$ & $T_{\mathrm{s}},{ }^{\circ} \mathrm{C}$ & $\begin{array}{c}(d T / d t) \mathrm{s} \\
{ }^{\circ} \mathrm{C} / \mathrm{s}\end{array}$ & \\
\hline 101001B1 & 1279.9 & 1148.9 & 1141.3 & -1.67 & 1135.6 & 1142.4 & 0.97 & 1150.9 & 1091.9 & -2.50 & 82 \\
\hline 101401B1 & 1273.8 & 1147.3 & 1141.6 & -1.04 & 1137.4 & 1140.0 & 0.58 & 1146.7 & 1082.4 & -2.54 & 86 \\
\hline 101402B 1 & 1256.1 & 1140.0 & 1139.3 & -0.33 & 1138.7 & 1140.0 & 0.34 & 1145.1 & 1082.4 & -2.34 & 87 \\
\hline 101403B1 & 1226.4 & 1141.5 & 1140.8 & -0.21 & 1139.9 & 1142.4 & 0.62 & 1148.5 & 1091.8 & -3.05 & 78 \\
\hline 101404B1 & 1283.2 & 1145.6 & 1140.0 & -0.76 & 1139.1 & 1143.2 & 0.82 & 1151.9 & 1096.6 & -2.58 & 80 \\
\hline 101701B1 & 1286.5 & 1140.7 & 1139.6 & -0.25 & 1139.3 & 1141.3 & 0.22 & 1145.5 & 1085.1 & -2.50 & 81 \\
\hline 101702B1 & 1304.4 & 1144.2 & 1140.4 & -1.04 & 1136.4 & 1141.3 & 0.74 & 1149.3 & 1086.9 & -2.46 & 76 \\
\hline 101703B 1 & 1285.0 & 1154.0 & 1146.8 & -1.43 & 1139.1 & 1140.4 & 0.42 & 1146.8 & 1087.8 & -2.78 & 84 \\
\hline 101704B1 & 1285.3 & 1146.0 & 1143.1 & -0.96 & 1139.4 & 1145.0 & 0.66 & 1150.8 & 1086.9 & -2.26 & 82 \\
\hline 101801B1 & 1265.6 & 1145.6 & 1141.2 & -0.76 & 1139.9 & 1143.1 & 0.50 & 1150.8 & 1094.3 & -2.54 & 85 \\
\hline 101802B1 & 1289.5 & 1143.4 & 1141.3 & -0.17 & 1140.9 & 1145.0 & 0.58 & 1150.8 & 1094.3 & -2.70 & 83 \\
\hline 102101B1 & 1266.1 & 1156.4 & 1146.7 & -1.55 & 1138.8 & 1141.3 & 0.58 & 1149.4 & 1091.6 & -2.74 & 76 \\
\hline 102102B 1 & 1288.1 & 1150.6 & 1143.1 & -1.39 & 1137.9 & 1143.1 & 0.86 & 1152.2 & 1093.3 & -2.10 & 80 \\
\hline 102801B1 & 1273.8 & 1151.4 & 1145.0 & -1.47 & 1138.5 & 1139.4 & 0.22 & 1142.8 & 1086.9 & -2.90 & 85 \\
\hline 103101B1 & 1235.0 & 1145.5 & 1140.0 & -1.12 & 1136.0 & 1138.4 & 0.46 & 1144.0 & 1081.6 & -3.17 & 90 \\
\hline 103102B 1 & 1317.5 & 1146.2 & 1143.2 & -0.80 & 1141.5 & 1147.9 & 0.86 & 1154.7 & 1087.9 & -2.18 & 88 \\
\hline 110101B1 & 1313.1 & 1135.1 & 1135.1 & 0.14 & 1135.1 & 1140.0 & 0.50 & 1149.6 & 1083.9 & -2.10 & 72 \\
\hline 110102B1 & 1319.9 & 1139.4 & 1137.7 & 0.11 & 1136.3 & 1144.0 & 0.82 & 1152.4 & 1087.1 & -2.02 & 86 \\
\hline 110103B1 & 1312.0 & 1140.8 & 1140.0 & -0.25 & 1140.3 & 1144.7 & 0.58 & 1151.7 & 1092.7 & -2.34 & 87 \\
\hline 110201B1 & 1305.1 & 1144.9 & 1141.6 & -0.44 & 1140.8 & 1144.0 & 0.62 & 1151.9 & 1091.1 & -2.15 & 79 \\
\hline 110202B1 & 1317.2 & 1148.8 & 1141.6 & -0.96 & 1138.7 & 1144.7 & 0.97 & 1153.5 & 1099.0 & -2.10 & 75 \\
\hline 110203B1 & 1260.4 & 1144.6 & 1140.4 & -0.60 & 1139 & 1144.0 & 0.97 & 1152.6 & 1097.9 & -2.42 & 85 \\
\hline $\begin{array}{c}\text { Mean values } \\
\qquad P_{R i}\end{array}$ & 1283.8 & 1145.5 & 1141.4 & -0.77 & 1138.6 & 1142.5 & 0.63 & 1149.6 & 1089.7 & -2.48 & 82 \\
\hline
\end{tabular}

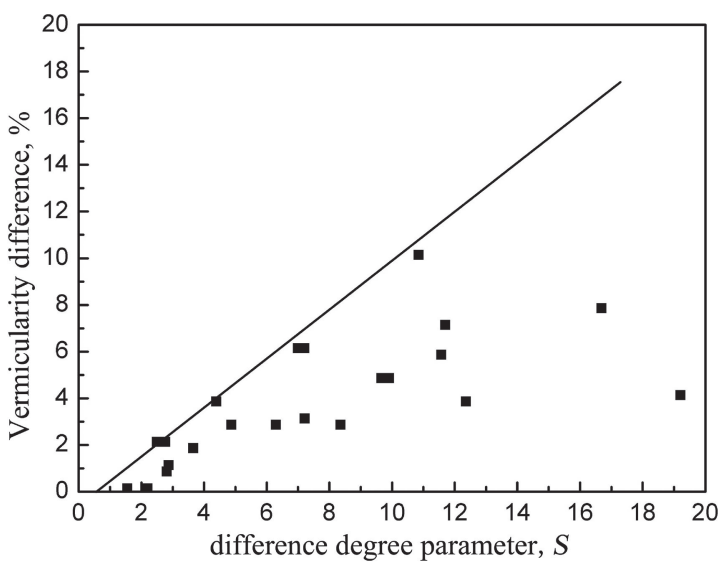

Fig. 3 The relationship of the difference degree parameter $S$ and vermicularity difference of CGI: no obvious regression relationship between them while the vermicularity difference approaches to zero with the decreasing of $S$

the vermicularity of CGI and the multiple characteristic parameters of the hypothetical ideal molten iron state. However, the trajectory trend of the envelope line showed when the difference degree parameter $S$ tends to zero, the vermicularity difference tends to the zero convergence. The result is consistent with the results of the cooling curve pattern recognition method. ${ }^{11-14)}$ It showed that when the difference degree parameter $S$ is small enough, the metallurgical quality state of the measured molten iron is close to the ideal state under the production conditions, thus it can be concluded that the vermiculation effect of the molten iron is qualified. Therefore, when the sample capacity of the cooling curve for the hypothetical ideal metallurgical quality state establishment is large enough, the threshold value for judging the different vermicularity of the molten iron can be determined, so as to realize the online regulation and control by this multiple characteristic point thermal analysis mothed.

Figure 4 shows two cooling curves of vermicularized molten iron with difference degree $\mathrm{S}$ of 8.3 and 30 , and the corresponding microstructure. As shown in Fig. 4, the flake graphite occurs corresponding to the molten iron with a difference degree $S$ of 30 . But the vermicularity $V_{\mathrm{G}}$ of the molten iron with difference degree $S$ of 8.3 is still as high as $84 \%$, and no flake graphite can be seen. In conclusion, When the difference of comprehensive metallurgical state of the molten iron reaches a certain value, the vermiculation effect of the corresponding molten iron will change significantly. 

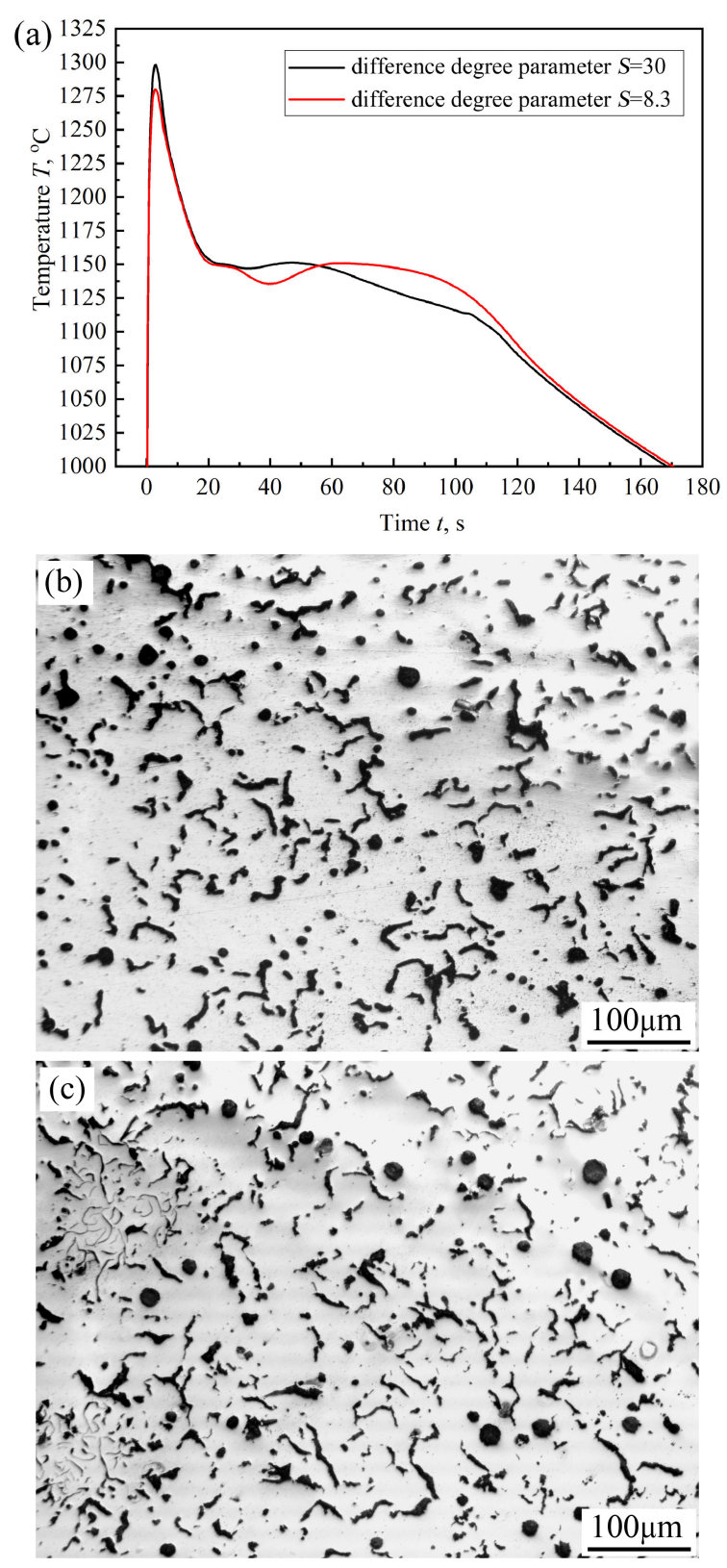

Fig. 4 Cooling curves with different $S$ calculated by 10 characteristic points and corresponding graphite morphology of sample center: (a) Two typical cooling curves with $S=8.3$ and 30 , (b) $S=8.3, V_{\mathrm{G}}=84 \%$, (c) $S=30$, flake graphite occurrence.
Therefore, the evaluation method of vermiculation effect of CGI by multi-characteristic point thermal analysis can meet the evaluation requirements.

\section{Conclusions}

(1) There is no direct correlation between the vermiculation effect and a single characteristic point of cooling curve, but there is statistical correlation between the vermiculation effect and multiple characteristic points.

(2) On the basis of analysis of multiple characteristic points representing the cooling curve characteristics, a multicharacteristic point representation model of hypothetical ideal metallurgical quality state of molten iron was established, which can represent the best state of production conditions, and can be used as a reference standard for judging the metallurgical state of the measured molten iron.

(3) The multi-characteristic point representation model can meet the requirements of evaluating the vermiculation effect.

\section{REFERENCES}

1) R.W. Gregorutti and J.E. Grau: Int. J. Cast Met. Res. 27 (2014) 275281.

2) G. Alonso, D.M. Stefanescu, P. Larranaga and R. Suarez: Int. J. Cast Met. Res. 29 (2016) 2-11.

3) C.H. Lim and B.C. Goo: Met. Mater. Int. 17 (2011) 199-205.

4) S. Dawson: China Foundry 6 (2009) 241-246.

5) J.C. Pina, S. Shafqat, V.G. Kouznetsova, J.P.M. Hoefnagels and M.G.D. Geers: Mater. Sci. Eng. A 658 (2016) 439-449.

6) T. Hara, T. Kitagawa, K. Kuroki, S. Saikawa, K. Terayama, S. Ikeno and K. Matsuda: Mater. Trans. 55 (2014) 1500-1505.

7) F. Mampaey, D. Habets, J. Plessers and F. Seutens: Int. J. Metalcast. 4 (2010) 25-43.

8) D.E. Anca, M. Chisamera, S. Stan and I. Riposan: J. Mater. Res. Technol. 8 (2019) 5160-5170.

9) A. Regordosa, U. Torre de la, A. Loizaga, J. Sertucha and J. Lacaze: Int. J. Metalcast. 14 (2020) 681-688.

10) J.H. Liu, L.T. Yi, G.L. Li, C.Q. Liu, Y.G. Li and Z.Y. Yang: China Foundry 8 (2011) 295-299.

11) X.J. Sun, Y.X. Li and X. Chen: J. Mater. Process. Technol. 200 (2008) $471-480$.

12) Z.H. Li and Y.X. Li: Metall. Mater. Trans. A 36 (2005) 2455-2460.

13) Y.X. Li and Q. Wang: J. Mater. Process. Technol. 161 (2005) 430-434.

14) X.J. Sun, Y.X. Li and X. Chen: Mater. Sci. Eng. A 466 (2007) 1-8. 